\title{
Electronic energy migration and rotation within bichromophoric molecules
}

\author{
Lennart B.-Å. Johansson and Jan Karolin \\ Department of Physical Chemistry, Umeå University, S-901 78 Umeå, Sweden
}

\begin{abstract}
Fluorescence anisotropy studies of donor-donor electronic energy is complicated by the influence of reorientational motions of the donor $(d)$ molecules. To test theoretical models for analyzing the fluorescence anisotropy, we have studied well-defined bichromophoric molecules, namely; a bis (9anthryl methyl phosphonate) bisteroid and the P3 Cys-P203 Cys double mutant of the latent plasminogen activator inhibitor 1 (PAI-1), labelled with sulphydryl specific derivatives BODIPY. A procedure is presented for extracting the rate of energy transfer, as well as the $d$-d distance from the fluorescence anisotropy.
\end{abstract}

\section{INTRODUCTION}

The Förster mechanism of electronic energy transfer ${ }^{1}$ has been studied and applied in a large number of papers. However, relatively few of them deal with transfer within bichromophoric molecules. Among such studies, most of them are devoted to donor-acceptor transfer, where the excitation is irreversibly transfered from the donor to the acceptor molecule $2-7$. Studies of donordonor $(d d)$ transfer, or migration between identical chromophores are even more rare, and usually vitrified samples are examined ${ }^{8}$. An likely reason for this is that also reorientation of the $d$ molecules will contribute to the fluorescence anisotropy, which is the necessary experiment for monitoring $d d$ migration.

In the present work, we have developed and experimentally tested a model for the fluorescence anisotropy which accounts for molecular reorientation and energy transfer. There are at least two motivations for studies of this kind. Firstly, a pure academic interest of being able to handle donor-donor transfer in the presence of molecular reorientation. Secondly and maybe a stronger reason, $d d$ transfer might serve as a tool for investigating structure of macromolecules, such as proteins and nucleic acids. Hitherto, donor-acceptor transfer has been frequently applied for this purpose ${ }^{9}$. The Förster mechanism predicts a rate of energy transfer which is inversely proportional to the sixth power of distance between the donor and acceptor molecules. The rate can be obtained from the fluorescence relaxation of the $d$ molecules, for example by means of single photon counting experiments. Thus knowing the rate of energy transfer, it is possible to calculate, or estimate the distance between the interacting $d$ molecules which could be located, for example, in a protein molecule. By means of site specific mutagenesis, it is in principle possible to replace any amino acid in a protein molecule by for example cystein. Cys is an interesting choice because a large number of sulfhydryl specific fluorescent probes are available ${ }^{10}$. This means that single, as well as double Cys mutants of a protein could be labelled by probes ${ }^{11-13}$. However, there is a big problem to achieve specific labelling of donor and acceptor molecules. In order to avoid this, we suggest using the same kind of fluorophore both as the donor and acceptor. However, unless the $d$ molecules of such a system are immobilized, the analysis of the anisotropy data will demand a model that accounts for both the rate of energy migration, as well as the reorientational correlation function. 


\section{EXPERIMENTAL}

The synthesis of the mono and bis(9anthrylmethylphosphonate) bisteroids is described in ref. 13. The procedure for preparing the BODIPY labelled Cys mutants of the plasmin activator inhibitor 1 (PAI-1) are given refs. 12 and 14. The sulphydryl specific probes, $\mathrm{N}$-((4,4-difluoro-5,7-dimethyl4-bora-3a,4a,-diaza-s-indacene-3-yl)methyl) iodoacetamide (SBDY) and N-(4,4-difluoro-5,7dimethyl-4-bora-3a,4a,-diaza-s-indacene-3-propionyl)N' -iodoacetylethylenediamine (LBDY) were purchased from Molecular Probes, Inc., Eugene, OR (USA).

The steady-state fluorescence spectra and anisotropies were obtained by using a SPEX Fluorolog 112 instrument (SPEX Industries, USA), equipped with Glan-Thompson polarizers. A PRA 3000 system (Photophysical Research Ass., Inc., Canada) was used for the polarized time-correlated single photon counting measurements of the fluorescence decay. Further details are given in refs. 13 and 14. Details for the analysis of data are given in ref. 15.

\section{Model}

\section{RESULTS and DISCUSSION}

The fluorescence anisotropy, $r(t)$, is an orientational correlation function that correlates the orientation of the excited molecules (given by eulerian angles $\Omega^{0}$ and $\Omega$ ) at the times of excitation $(t=0)$ and emission $(t=t)$. The rotatation of an excited molecule and the excitation-energy migration contribute to $r(t)$. We assume that the orientational correlation functions $\left\{r_{j}(t)\right\}$ and the excitation probability $\{p(t)\}$ of the two $d$ molecules can be separated, and that the fluorescence anisotropy can be written as:

$$
r(t)=\frac{1}{2}\left\{r_{1}(t)+r_{2}(t)\right\} p(t)+\frac{1}{2}\left\{r_{12}(t)+r_{21}(t)\right\}\{1-p(t)\}
$$

, Here $r_{i j}(t)$ denotes contributions due to energy migration from the initially excited $d_{i}$ molecule to its neighbor $d_{j}$. Note, the excitation probability is $p(t)$ for any donor being the initially excited one. Recently, the Liouville equation of energy transfer between a pair of coupled $d d$ molecules undergoing Brownian rotational motions was studied ${ }^{16}$. It was concluded that cross correlation between $r_{j}(t)$ and $p(t)$ is negligible, which supports the use of this assumption in eq. 1 .

For extracting molecular information from eq. 1, expressions for $r_{j}(t), r_{i j}(t)$ and $p(t)$ are needed. The rotational correlation function $r_{j}(t)$ is given by

$$
r_{j}(t)=\frac{2}{5} \sum_{m=-2}^{m=2}<D_{m 0}^{(2)}\left[\Omega_{L M_{j}}(0)\right] D_{-m 0}^{(2)}\left[\Omega_{L M_{j}}(t)\right]>(-1)^{m}
$$

The brackets $\langle\ldots\rangle$ denote orientational averages over the ensemble of $d$ molecules, and $D_{m 0}^{(2)}(\Omega)$ are second rank Wigner rotational matrices ${ }^{17}$. The subscripts $\mathrm{LM}_{j}$ indicate the transformation (see Fig. 1) from the laboratory $(\mathbf{L})$ to the molecular frame $(\mathbf{M j})$. The corresponding expression for $r_{i j}(t)$ reads:

$$
r_{i j}(t)=\frac{2}{5} \sum_{m=-2}^{m=2}<D_{m 0}^{(2)}\left[\Omega_{L M_{i}}(0)\right] D_{-m 0}^{(2)}\left[\Omega_{L M_{j}}(t)\right]>(-1)^{m}
$$

The averaging of eqs. 2 and 3 over an isotropic global orientation $\left(\Omega_{L R}\right)$ and a local uniaxial anisotropic ( $\Omega_{D_{i} M_{i}}$ ) distribution density function yields:

$$
r_{i}(t)=\frac{2}{5}\left[\left\{1-\rho_{i}^{\infty}\right\} \gamma(t)+\rho_{i}^{\infty}\right]
$$

and

$$
r_{i j}(t)=\frac{2}{5}\left[\left(\rho_{i j}^{0}-\rho_{i j}^{\infty}\right] \Gamma(t)+\rho_{i j}^{\infty}\right]
$$


where

$$
r_{i}\left(t_{\infty}\right)=\frac{2}{5}<D_{0 O}^{(2)}\left(\Omega_{D_{i} M_{i}}\right)>^{2} \equiv \frac{2}{5} \rho_{i}^{\infty}
$$

and

$$
r_{i j}\left(t_{\infty}\right)=\frac{2}{5} D_{O 0}^{(2)}(\delta)<D_{O 0}^{(2)}\left(\Omega_{D_{i} M_{i}}\right)><D_{O 0}^{(2)}\left(\Omega_{D_{j} M_{j}}\right)>\equiv \frac{2}{5} \rho_{i j}^{\infty}
$$

where $\gamma(t)$ and $\Gamma(t)$ are taken to be a sum of exponential functions. More details for this model are given in ref. 15.
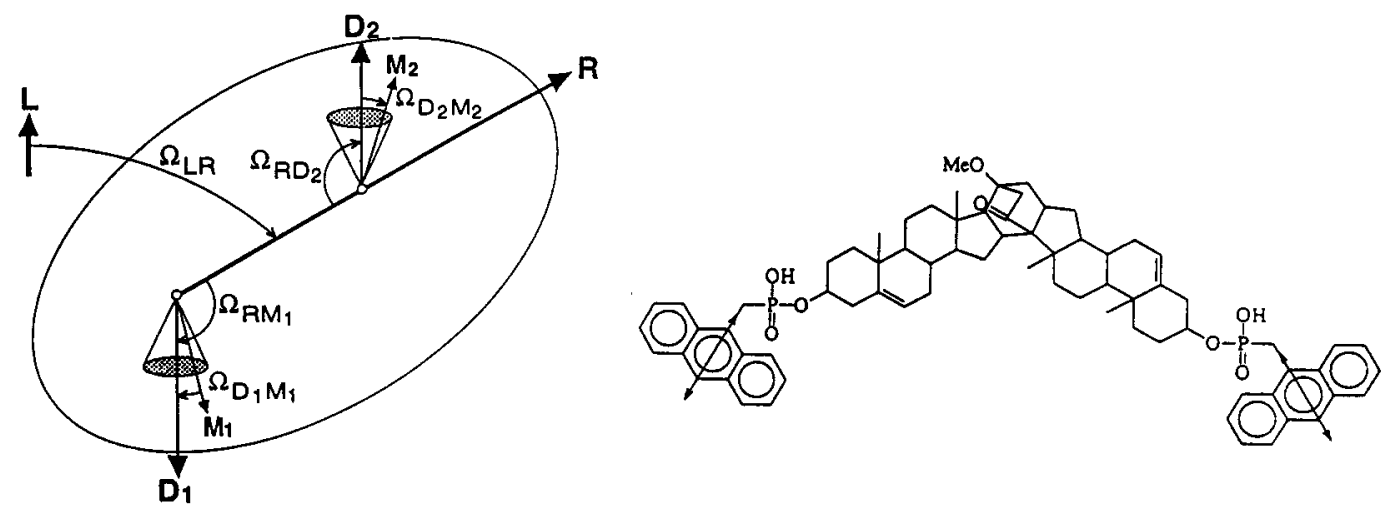

Figure 1. The schematic illustrates the different frames involved in describing energy transfer within a bichromophoric molecule. Here $\mathbf{R}$ denotes a molecule fixed frame. The donor molecules (denoted as number 1 and 2) are uniaxially oriented about the $z$-axes of the $D_{1}$ and $D_{2}$ frames. The $\Omega$ denote the Euler angles, $\Omega$ provides the orientational transformations between the laboratory $(L), R, D_{i}$ and molecular $\left(\mathbf{M}_{\mathbf{i}}\right)$ frames. The structure formula shows the bis(9-anthrylmethylphosphonate) bisteroid. The arrows indicate the electronic transition dipole moment.

In the dynamic (and static) limit the master equation of energy transfer is readily solved for a dd pair separated at a fixed distance. The probability that the initially excited donor is still excited a time $t=t$ later is given by ${ }^{1}$

$$
p(t)=\frac{1}{2}(1+\exp (-2 \omega t))
$$

The rate of energy migration $(\omega)$ depends on the average value of $\kappa^{2}$. In the dynamic limit, < $\kappa^{2}$. can be expressed in terms of averaged Wigner rotational matrices, or order parameters according to

$$
\begin{aligned}
& =\kappa^{2}>=\frac{2}{3}+\frac{2}{3} D_{00}^{(2)}(\delta)<D_{00}^{(2)}\left(\Omega_{D_{i} M_{i}}\right)><D_{00}^{(2)}\left(\Omega_{D_{j} M_{j}}\right)>+\frac{2}{3}\left(D_{00}^{(2)}\left(\Omega_{R D_{i}}\right)<D_{00}^{(2)}\left(\Omega_{D_{i} M_{i}}\right)>+\right. \\
& \left.+D_{00}^{(2)}\left(\Omega_{R D_{j}}\right)<D_{00}^{(2)}\left(\Omega_{D_{j} M_{j}}\right)>\right\}+\frac{4}{3} D_{00}^{(2)}\left(\Omega_{R D_{i}}\right) D_{00}^{(2)}\left(\Omega_{R D_{j}}\right)<D_{00}^{(2)}\left(\Omega_{D_{i} M_{i}}\right)><D_{00}^{(2)}\left(\Omega_{D_{j} M_{j}}\right)>- \\
& -6 \sin \beta_{R D_{i}} \cos \beta_{R D_{i}} \sin \beta_{R D_{j}} \cos \beta_{R D_{j}} \cos \alpha_{R D_{j i}}<D_{00}^{(2)}\left(\Omega_{D_{i} M_{i}}\right)><D_{00}^{(2)}\left(\Omega_{D_{j} M_{j}}\right)>
\end{aligned}
$$

The angle between the symmetry axis $\left(D_{\mathfrak{i}}\right)$ of the local orientational distributions of the donors $d_{1}$ and $d_{2}$ is denoted by $\delta$.

\section{Applications}

Two different systems were used for testing the model. The first system consists of a bisteroid to which either one or two anthrylmethylphosphone groups are covalently linked. The bisteroid 
serves as a rigid link between the anthracenes. From the molecular structure, the distance between the anthracenes was obtained to be $24.2 \AA^{15}$. The second system consists of three Cys mutants of PAI-1 in its latent from, namely; P3 Cys, P203 Cys and P3 Cys-P203 Cys. The mutants were covalently linked with two different sulphydryl specific BODIPY derivatives, here denoted by SBDY and LBDY. From the X-ray structure ${ }^{18}$, one obtains a distance of $46.8 \AA$ between the $C_{\alpha}$ carbons of $\mathrm{P} 3$ and $\mathrm{P} 203$ amino acids.

The experimental strategy was to determine the fluorescence anisotropy of both the mono- and bichromophoric forms of the two systems, and to combine them in a global analysis, respectively. The fluorescence anisotropy of the mono forms contains information solely about the orientational correlation function (eqs. 4 and 6), while the $d d$ form will depend on both the transfer rate and reorientation. The fluorescence steady-state anisostropy $\left(r_{s}\right)$ obtained for both systems are summarized in Table 1. For bichromophoric molecules, the $r_{s}$ values are always smaller as compared to the arithmetic mean value $\left(\left\langle r_{s}\right\rangle\right)$ of corresponding mono forms. This shows that in addition to reorientation, there must be an additional depolarizing process, which is $d d$ energy migration.

TABLE 1. Results from global analysis of the time-resolved fluorescence anisotropy obtained for mono and bis-(9anthrylmethylphosphonate), and the P3 Cys, P203 Cys and P3 Cys - P203 Cys mutants of latent PAI-1 labelled with SBDY and LBDY

\begin{tabular}{|c|c|c|c|c|c|c|c|c|}
\hline System & Form & $r_{s}$ & $\phi / \mathrm{ns}$ & $\rho^{0}$ & $\omega \times 10^{-9} / \mathrm{s}^{-1}$ & $\left\langle x^{2}\right\rangle$ & $\delta /^{\circ}$ & $R / \AA$ \\
\hline $\begin{array}{l}\text { Bisteroid in1,2-propanediol } \\
\text { at } 288 \mathrm{~K}\end{array}$ & $\begin{array}{l}\text { mono } \\
\text { bis }\end{array}$ & $\begin{array}{l}0.141 \\
0.103\end{array}$ & $7.5 \pm 1.0$ & 0.275 & $0.45 \pm 0.1$ & 1.05 & 129.2 & $23.2 \pm 2$ \\
\hline $\begin{array}{l}\text { Latent PAI- } 1 \text { in } \\
\mathrm{H}_{2} \mathrm{O}: \text { glycerol } \\
50: 50 \% \mathrm{w} / \mathrm{w} \text { at } 277 \mathrm{~K}\end{array}$ & $\begin{array}{l}\text { P3-SBDY } \\
\text { P203-SBDY } \\
\text { P3-P203-SBDY }\end{array}$ & $\begin{array}{l}0.273 \\
0.310 \\
0.226\end{array}$ & $\begin{array}{l}6.9 \pm 1.6 \\
12.3 \pm 2.8\end{array}$ & 0.60 & $1.84 \pm 0.11$ & 1.45 & $48.2^{a}$ & $45.4 \pm 2$ \\
\hline $\begin{array}{l}\text { Latent PAI- } 1 \text { in } \\
\mathrm{H}_{2} \mathrm{O}: \text { glycerol } \\
50: 50 \% \mathrm{w} / \mathrm{w} \text { at } 277 \mathrm{~K}\end{array}$ & $\begin{array}{l}\text { P3-LBDY } \\
\text { P203-LBDY } \\
\text { P3-P203-LBDY }\end{array}$ & $\begin{array}{l}0.251 \\
0.248 \\
0.199\end{array}$ & $\begin{array}{l}7.7 \pm 1.0 \\
6.8 \pm 1.0\end{array}$ & 0.55 & $1.08 \pm 0.17$ & 1.45 & $42.0^{a}$ & $50.8 \pm 2$ \\
\hline
\end{tabular}

In the global analysis of the bisteroid data, the values on $\phi, \rho^{\circ}$ and the limiting anisotropy $\left(r_{0}=\right.$ $2 / 5$, ideally) were simultanously fitted to the time-resolved anisotropy obtained for the mono and bis forms. The rate of energy migration and $\rho^{0}$ were treated as floating parameters. The angle between the symmetry axes $(\delta)$ were either treated as a floating, or as a fixed, parameter. The values obtained for the distance $(R)$ between the anthracence moieties, as well as $\delta$ are in excellent agreement with the known values of $24.2 \AA$ and $128^{\circ}, 15$. Moreover, the value on $\rho^{\circ}$ is acceptable since it can be shown that $0 \leq \rho^{0} \leq 1$.

In the global analysis of the labelled PAI-1 mutants, the values on $\phi\left(d_{1}\right), \phi\left(d_{2}\right)$ and $r_{0}$ were simultanously fitted to $r(t)$ obtained for the two single mutants, and the double mutant. The parameters $\rho^{0}, \omega$ and $\delta$ were treated as floating parameters. Typical results obtained by fitting the model to the data are shown in Fig. 2. The distance between the $d d$ pair can be calculated from the rate of energy migration $\left[\omega=1.5\left\langle\kappa^{2}\right\rangle \tau^{-1}\left(R_{0} R^{-1}\right)^{6}\right.$, where $\tau$ is the fluorescence lifetime] provided $\left\langle\kappa^{2}\right\rangle$ (see eq. 9) is known. For estimating $\left\langle\kappa^{2}\right\rangle$, the orientational distribution of the donor molecule having the lowest local order (i.e. the lowest value on $\rho^{\infty}$ ) was approximated to be effectively uniaxial about $R$ (see Fig. 1). The calculated distances between the BODIPY groups are in good agreement with the $X$-ray data (See Table 1), although neither of the donors could be located at the positions of the $\mathrm{C}_{\alpha}$ atoms of $\mathrm{P} 3$ and P203. A more extensive analysis will be given in a forthcoming paper.

The model for describing the fluorescence anisotropy works very well for extracting the rate of energy migration, as well as the distance between interacting donor molecules. The reason why the dynamic approximation seems to be justified is not evident. Strictly speaking the dynamic approximation means that $\omega \ll 1 / \phi$, which is not the case in this study. However, one should 

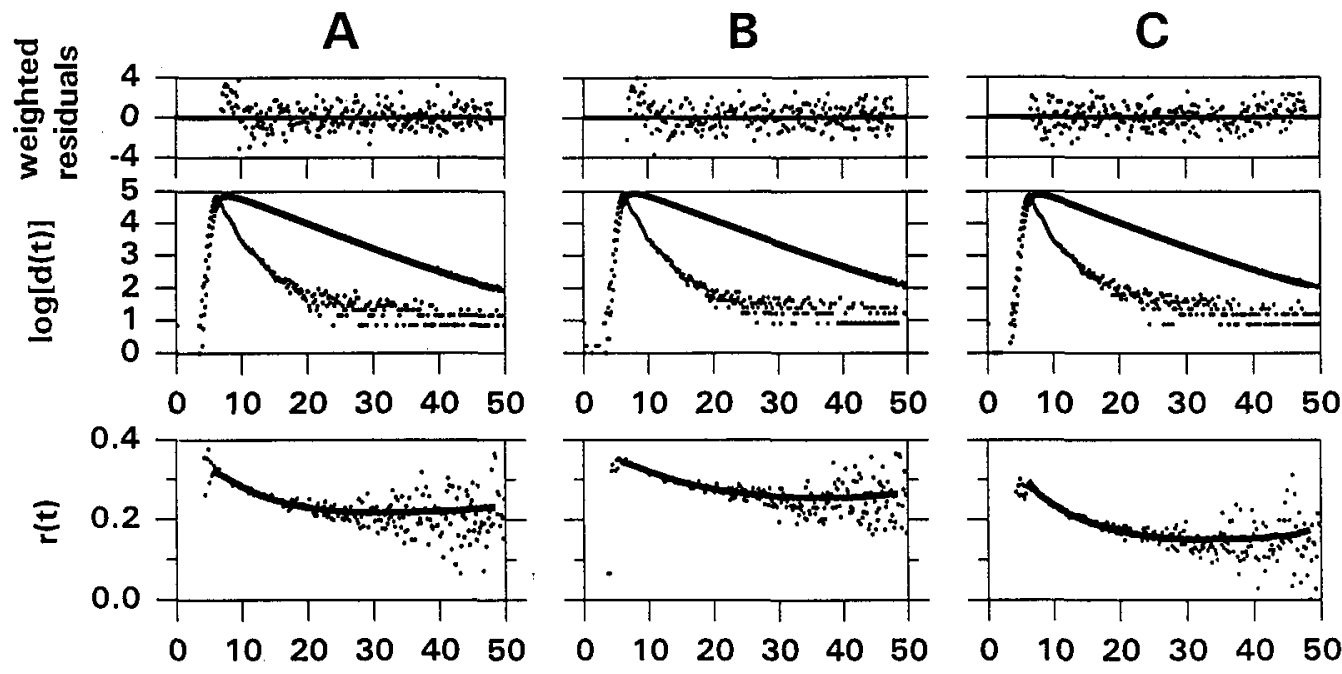

time/ns

Figure 2. Time-resolved fluorescence anisotropy $[r(t)]$ curves and difference $[d(t)]$ curves obtained for P3 Cys (A), P203 (B) and P3 Cys - P 203 Cys (C) mutants labelled with LBDY. The instrumental response function is shown with the $d(t)$ curves. The upper weighted residuals were obtained from the global analysis.

notice that in present study the initial anisotropies $\{r(0)\}$ found (data not shown) are always less than the limiting anisotropy. Indeed, this means that rapid unresolved motions of the donor moieties do occur at rates much faster than $\omega$. Hence, it might be that the presence of these rapid motions make the dynamic approximation acceptable, and/or that deviations between static and dynamic models in practise are small, although they differ substantially regarding their analytical complexity. This question is currently being studied and the present model is tested against more elaborate theories based on solving the stochastic Liouville equation.

\section{REFERENCES}

1. Th. Förster, Th., Ann. Physik, 2, 55 (1948).

2. S. A. Latt, H. T. Cheung, and E. R. Blout, J. Am. Chem. Soc., 87, 995 (1965).

3. B. Valeur, B. in Fluorescent Biomolecules. Methologies and Applications. pp. 269-303, D. M. Jameson and G. D. Reinhart Eds., Plenum Press, New York, (1989).

4. B. Valeur, J. Mugnier, J. Pouget, J. Bourson and F. Santi, J. Phys. Chem, 87, 6073 (1989).

5. M. Kaschke, N. P. Ernsting, B. Valeur, and J. Bourson, J. Phys. Chem., 94, 5757 (1990).

6. M. N. Berberan-Santos, B. Valeur, J. Chem. Phys., 95, 8048 (1991).

7. M. N. Berberan-Santos, J. Pouget, B. Valeur, J. Canceill, L. Jullien, and J.-M. Lehn, J. Phys. Chern., 97,11379 (1993).

8. T. Ikeda, B. Lee, S. Kurihara, S. Tazuke, S. Ito, and M. Yamamoto, J. Am. Chem. Soc., 110, 8299 (1988).

9. Van Der Meer, B., Coker III, G. and Chen, S.-Y. S, Resonance Energy Transfer, VCH Publishers, Inc., New York, (1994).

10. R. P. Haugland, Handbook of Fluorescent Probes and Research Chemicals, Molecular Probes, Eugene, OR, 5th Ed. (1992).

11. J. J. H. Lakey, D. Baty and F. Pattus, J. Mol. Biol., 218, 639 (1991).

12. L. Strandberg, J. Karolin, L. B.-A. Johansson, , M. Fa, S. Aleshkov, and T. Ny, Trombosis Research, 76, 253 (1994).

13. I. V. Grechishnikova, L. B.-A. Johansson and J. G. Molotkovsky, Chem. Phys, Lipids, In press (1996).

14. J. Karolin, L. B.-A. Johansson, L. Strandberg and T. Ny, J. Am. Chem. Soc., 116, 7801 (1994).

15. L. B.-A. Johansson, F. Bergström, P. Edman, I. V. Grechishnikova and J. G. Molotkovsky, J. Chem. Soc., Faraday Trans., 92, 1563 (1996).

16. I. Fedchenia, and P.-O. Westlund, Physical Review E, 50, 555 (1994).

17. D. M. Brink, and G. R. Satchler, Angular Momentum, 3rd ed., Clarendon Press, Oxford, (1993).

18. J. Mottonen, A. Strand, J. Symersky, R. M. Sweet, D. E. Danley, K. F. Geoghegan, R. D. Gerard and E. J. Goldsmith, Nature, 355, 270 (1992). 